15

\title{
Улучшение подъемных характеристик крыла со сверхкритическим профилем при использовании модулированного импульсного струйного актуатора
}

\author{
(C) Soheila Abdolahipour ${ }^{1}$, Mahmoud Mani ${ }^{2}$, Arash Shams Taleghani, ${ }^{1,}$ \\ ${ }^{1}$ Aerospace Research Institute, Ministry of Science, Research and Technology, Tehran, Iran \\ ${ }^{2}$ Department of Aerospace Engineering, Amirkabir University of Technology, Tehran, Iran \\ 『 E-mail: Arash.taleghani@gmail.com
}

Поступило в Редакцию 20 августа 2021 г.

В окончательной редакции 11 сентября 2021 r.

Принято к публикации 12 сентября 2021 г.

В результате экспериментального исследования предложена импульсная система управления потоком на устройство увеличения подъемной силы крыла с несущей поверхностью модели NASA SC(2)-0714 в диапазоне чисел Рейнольдса, соответствующих фазам взлета и приземления. Применен инновационный метод модуляции сигнала, обеспечивающий возможность одновременного использования достоинств как низких, так и высоких частот возбуждения в одном управляющем сигнале актуатора, которые считаются эффективными при управлении срывом потока. Обнаружено, что благодаря применению модулированных импульсных струй можно увеличивать коэффициенты подъемной силы и лобового сопротивления по сравнению с соответствующими значениями для простой импульсной струи.

Ключевые слова: управление потоком, конфигурация с высокой подъемной силой, импульсная струя, импульсная модуляция, аэродинамическая эффективность.

DOI: 10.21883/PJTF.2022.01.51869.18999

В последние годы для улучшения аэродинамических характеристик несущих поверхностей крыльев [1-4], устройств для увеличения подъемной силы $[5,6]$, дельтовидных крыльев [7], воздушных турбин [8-10] и лопаток турбомашин [11] широко использовались активные методы управления потоком, такие как обдувка, синтетические струи, плазменные актуаторы и пр. Цель систем управления потоками на несущие поверхности и крылья состоит в том, чтобы устранить или ослабить отрыв потоков, который обычно ускоряется при наличии положительного градиента давления. Большинство способов устранения срыва потока основано на трех методах: прямая подача высокоимпульсного потока в приповерхностную область, усиление смешивания и перераспределение импульса в пограничном слое за счет конвективных вихрей вдоль потока [12].

Эффективность управления воздушной обдувкой может быть существенно увеличена, если использовать импульсную струю вместо непрерывной. Первоначально считалось, что преимущество использования импульсной струи для управления отрывом потока состоит в экономии массового расхода на протяжении определенного периода, но дальнейшие исследования показали, что в импульсном режиме стимулируются образование дополнительных вихрей и поступление периодических вихревых структур на срывающийся пограничный слой [13]. За счет этих вихревых структур улучшается турбулентное перемешивание между низкоимпульсным приповерхностным пограничным слоем и внешней частью пограничного слоя в высокоимпульсном поперечном по- токе. Поэтому импульс передается только внутри самого потока, и не только от актуатора системы управления потоком. В результате оказывается возможным снизить требования к массовому потоку и энергопотреблению.

С другой стороны, предыдущие исследования показали, что наиболее эффективное управление отрывом потока достигается при частоте возбуждения, принадлежащей к диапазону естественных частот неустойчивых колебаний сорвавшегося сдвигового (пограничного) слоя. Литературные данные отчетливо показывают, что стратегия организации взаимосвязи между периодическим возбуждением и неустойчивостью потока приводит к значительному повышению эффективности управления потоком [13]. Для сорвавшегося потока, поступающего на подъемные поверхности (несущие поверхности), характерны два доминирующих вида неустойчивости: свернутые вихри в сдвиговом слое и крупномасштабные вихри в сорвавшейся с несущей поверхности вихревой дорожке [14]. Низкочастотное возбуждение создает крупные вихревые структуры, которые конвектируют вниз по потоку и усиливают перемешивание потока, в результате которого происходит обратное присоединение потока [15]. В то же время при высокочастотном возбуждении образуются меньшие по размеру вихри, приводящие к видимому изменению аэродинамической формы поверхности. Эта модификация приводит к соответствующим изменениям градиента давления и, следовательно, к подавлению отрыва потока [16]. Частоты возбуждения, соответствующие этим двум подходам, различаются по меньшей мере на порядок, составляя, 
как правило, $\mathrm{O}(1)$ и $\mathrm{O}(10)$ соответственно для низких и высоких частот.

В настоящей работе для улучшения аэродинамической эффективности устройств для повышения подъемной силы применялся новый метод модуляции импульсной струи, приводящий к снижению расхода подаваемого воздуха. Для этого использовался струйный актуатор определенного типа, позволяющий организовать одновременную подачу высокоимпульсного приповерхностного потока и генерацию вихрей вдоль размаха крыльев и вдоль потока, обеспечивающих перераспределение импульса в пограничном слое и усиление перемешивания. В рамках данного метода форма сигнала возбуждения изменяется благодаря введению импульсной модуляции, что делает возможным одновременное использование достоинств высоких (несущий сигнал) и низких (модулирующий сигнал) частот возбуждения. Модулированный сигнал возбуждения уже применялся раньше во многих системах управления потоком, таких как плазменный актуатор [17-19] и синтетическая струя [20], но в настоящей работе метод импульсной модуляции был впервые использован для сигнала возбуждения импульсной струи, создаваемой быстродействующим электромагнитным клапаном и имеющей форму прямоугольной волны. Обзор литературы показал, что модулированное возбуждение заметно изменяет аэродинамические характеристики по сравнению со случаем простых синусоидальных сигналов в актуаторах плазменной и синтетической струи (в лабораторном масштабе), однако из-за низкого импульса его подачи устройства управления потоком становятся неприменимыми в промышленных приложениях с повышенными требованиями к надежности.

В настоящей работе для исследования была выбрана двумерная модель крыла с несущей поверхностью типа NASA SC(2)-0714. Модель крыла была построена в виде двухкомпонентной конфигурации с высокой подъемной силой, состоящей из основного элемента и однощелевого закрылка с закругленным краем с соотношением длин хорд 1/4. Аэродинамическая труба была рассчитана на размах крыльев $1.5 \mathrm{~m}$ при длине хорды $0.6 \mathrm{~m}$.

В этих экспериментах импульсные струи вводились через систему актуатора в поток на стороне всасывания закрылка. Система актуатора была оборудована шестнадцатью электромеханическими быстродействующими клапанами, трубопроводом для сжатого воздуха и программируемым логическим контроллером (ПЛК). Каждый клапан представлял собой блок промышленного изготовления (FESTO MHJ9-QS-4-MF), рассчитанный на эксплуатацию в диапазоне входных давлений 0.5-6.0 bar. Стандартный номинальный расход клапана составлял 100 1/min. Через систему ПЛК программировались такие рабочие параметры клапана, как состояние вкл/выкл, длина рабочего цикла (заполнение цикла) и частота пульсации. Система ПЛК позволяет генерировать модулированный импульсный сигнал.

Сжатый воздух подавался на клапаны по трубам через компрессор. Амплитуда импульсов задавалась стандарт- ным регулятором давления, установленным перед выходом компрессора на фильтр, давление воздуха устанавливалось равным 6 bar. Затем трубопровод отводился к клапанам через патрубок. Выходы клапанов подключались к камерам осаждения таким образом, чтобы каждый клапан подавал воздух в свою собственную камеру. Каждая камера была закрыта крышкой, в которой были проделаны две щели. Расположение щелей было рассчитано в соответствии с формой генератора вихрей расходящейся конфигурации (с общим стоком). Массив пар щелей распределялся вдоль плеча закрылка на расстоянии, составляющем $40 \%$ от длины хорды закрылка. Выходной поток каждой щели не только обеспечивает непосредственное формирование импульсов, но и создает вихрь.

Все актуаторы работали в фазе, а скорость струи соответствовала управляющим сигналам клапанов. Чтобы убедиться в корректности функционирования устройства и правильности значения напряжения, подаваемого на клапаны, напряжение системы возбуждения выборочно проверялось с помощью осциллографа. На рис. 1, $a$ представлена форма импульсов входного напряжения клапанов, отображаемых на осциллографе, в режиме модуляции при несущей частоте $100 \mathrm{~Hz}$ и частоте модуляции $10 \mathrm{~Hz}$. Данные осциллографа подтвердили, что устройство генерирует сигналы с необходимой точностью. Кроме того, на рис. $1, b$ показана зависимость от этого возбуждения выходной скорости струи от одного актуатора (на расстоянии $50 \mathrm{~mm}$ от выхода клапана по продольной оси), измеренная с помощью термоэлектрического анемометра 55p11 Dantec. Мгновенная скорость струи может достигать примерно $30 \mathrm{~m} / \mathrm{s}$. Эта величина - мгновенная скорость струи - в какой-то степени отображает влияние модуляции импульсов на интенсивность турбулентности и флуктуации значения скорости.

Значительный интерес вызывает колебательное изменение угла атаки при фиксированном отклонении закрылка, поскольку этот процесс можно рассматривать как модель поведения крыла при взлете и приземлении. Таким образом, угол отклонения закрылка (с закругленным краем) принимался равным $35^{\circ}$. Кроме того, была проведена серия экспериментов при скорости невозмущенного потока $V=25 \mathrm{~m} / \mathrm{s}$, которая при определенной длине хорды дала число Рейнольдса порядка $1.01 \cdot 10^{6}$. В настоящей работе для возбуждения потока использовались два типа импульсных струй: первый - простая импульсная струя (SPJ) с частотой возбуждения $250 \mathrm{~Hz}$ $\left(F^{+}=1.8, F^{+}=f L / V\right.$, где $f-$ частота возбуждения, $L$ - длина закрылка $(L=18 \mathrm{~cm})$, а $V$ - скорость невозмущенного потока); второй - модулированная импульсная струя (MPJ). Модулированные импульсные струи генерировались при несущей частоте $250 \mathrm{~Hz}\left(F^{+}=1.8\right)$ и частоте модуляции 10,30 и $45 \mathrm{~Hz}\left(F^{+}=0.07,0.22\right.$ и 0.32 соответственно).

В настоящей работе аэродинамические силы измерялись с помощью установленных снаружи трехкомпо- 

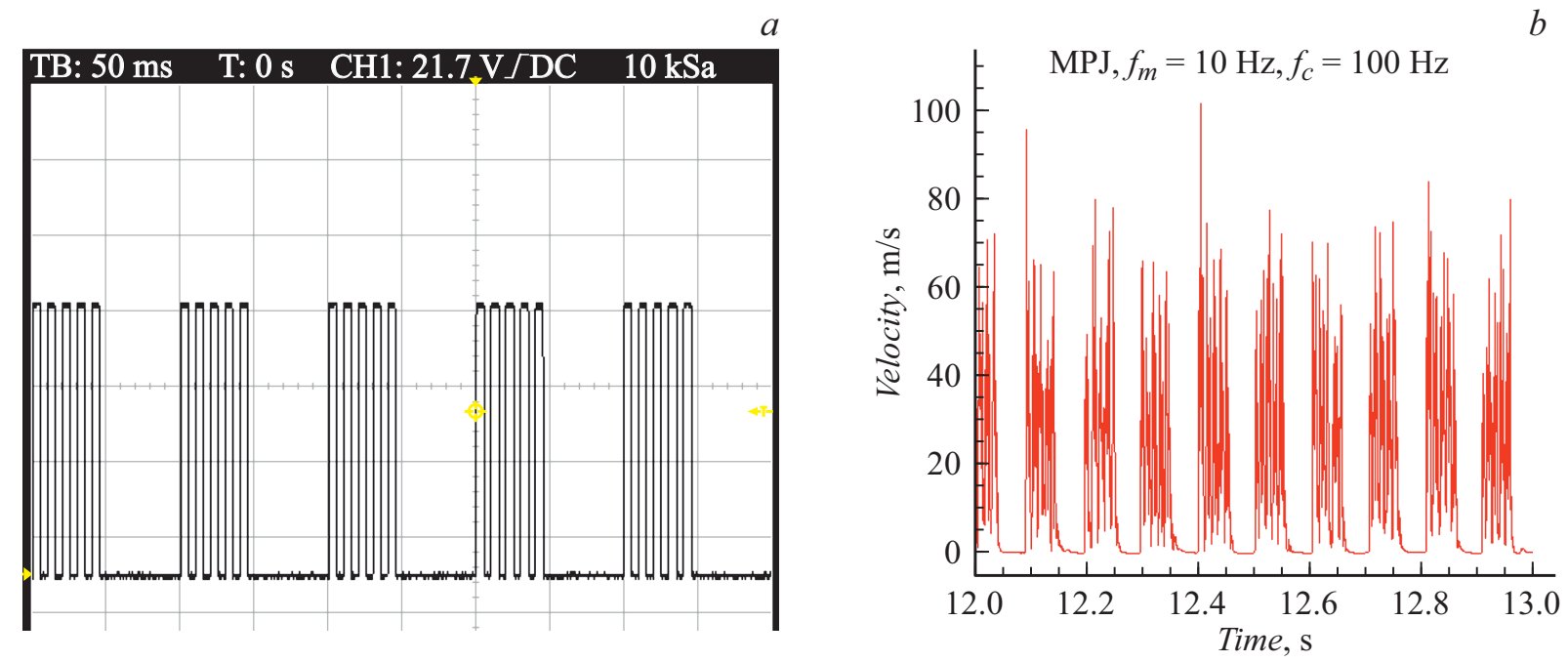

Рис. 1. Импульсная модуляция сигнала. $a$ - форма сигнала напряжения на осциллографе, $b-$ мгновенная скорость модулированной импульсной струи.
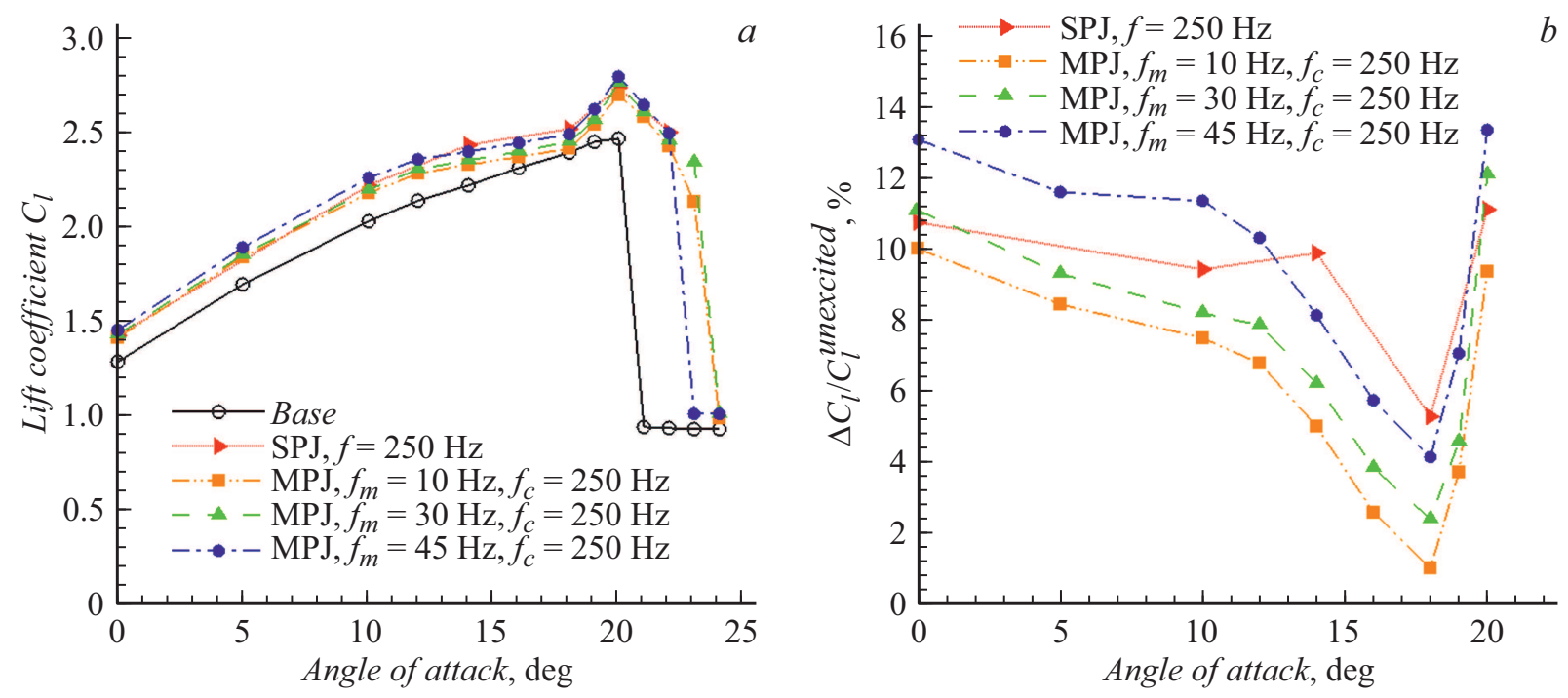

Рис. 2. Зависимости коэффициента $(a)$ и приращения $(b)$ подъемной силы от угла атаки при наличии и отсутствии возбуждения.

нентных тензометрических весов. На рис. 2, а представлены зависимости коэффициента подъемной силы от угла атаки для случаев наличия и отсутствия возбуждения потока. Из этого рисунка видно, что в случае отсутствия возбуждения при угле $21^{\circ}$ наблюдаются значительные потери в подъемной силе, что является условием срыва потока. Данные, полученные в случаях наличия возбуждения, показывают, что импульсные струи для всех четырех возбуждающих сигналов дают значительный рост коэффициентов подъемной силы для всех возможных углов атаки, однако в степени роста подъемной силы наблюдаются различия. Эти данные указывают также на то, что возбуждение импульсной струи теряет силу воздействия на поток при угле атаки 16-18. Причина может заключаться в том, что направление возбуждающих струй и направление потока через щели клапанов, а также их комбинации с невозбужденным потоком могут нежелательным образом изменяться при изменении угла атаки. При угле атаки 20॰ $\left(\alpha_{c l \max }\right)$ возбуждение импульсной струи приводит к задержке отрыва потока от поверхности закрылка и восстановлению подъемной силы до более высокого значения, чем в случае невозбужденного потока. Кроме того, в случае наличия возбуждения отрыв происходит не внезапно, что указывает на то, что даже в послесрывной области (при закритических углах атаки) система управления потоком продолжает играть заметную роль, и возбуждение импульсной струи может оказывать положительное влияние на процесс отрыва. Среди различных видов возбуждения больше преимуществ в отношении увеличения подъемной силы по сравнению со случаем возбуждения SPJ дает возбуждение MPJ при частоте 

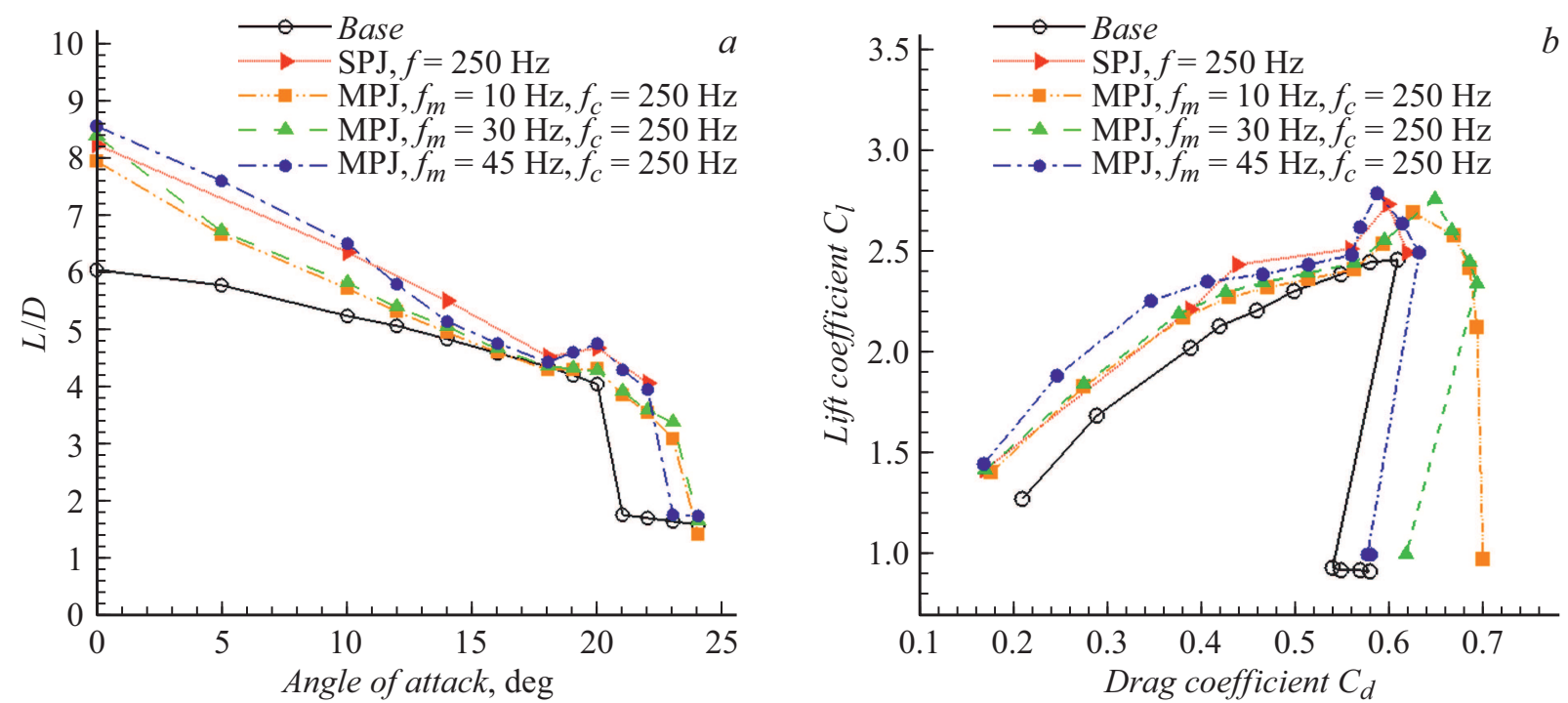

Рис. 3. Зависимость отношения между подъемной силой и силой лобового сопротивления от угла атаки $(a)$ и полярная диаграмма лобового сопротивления атаки $(b)$ при наличии и отсутствии возбуждения.

модуляции $45 \mathrm{~Hz}$. Вместе с тем при частоте модуляции от 10 до $30 \mathrm{~Hz}$ MPJ не давала значительного улучшения рабочих характеристик по сравнению со случаем модуляции с частотой $45 \mathrm{~Hz}$. Это значит, что, возможно, частота $45 \mathrm{~Hz}$ относится к первой субгармонике или гармонике естественных нарушений устойчивости (частоте вихревых дорожек в неуправляемом потоке). Это предположение согласуется с данными предыдущих экспериментальных исследований по влиянию частоты возбуждения на несущие поверхности крыльев, на которых происходит срыв пограничного слоя. В большинстве исследований, проведенных при различных числах Рейнольдса, в качестве оптимальной безразмерной частоты предлагалась величина $0.3<F^{+}<4$ [13].

На рис. 2, $b$ представлена зависимость относительного увеличения подъемной силы от угла атаки при различных возбуждающих сигналах. Из рис. 2, $b$ видно, что при малых углах атаки МРЈ при частоте модуляции $45 \mathrm{~Hz}$ являлась оптимальным сигналом возбуждения, при котором наблюдалось максимальное приращение подъемной силы. Оптимальным сигналом возбуждения при углах атаки 14 и $18^{\circ}$ была SPJ при частоте $250 \mathrm{~Hz}$. Однако при угле атаки $20^{\circ}$ MPJ опять обеспечивает максимальное увеличение подъемной силы, причем на величину, значительно превышающую ту, которая имеет место в случае SPJ. При угле атаки $20^{\circ}$ приращение подъемной силы составило $13.3 \%$ для МРЈ и $11.1 \%$ для SPJ.

Преимущество возбуждения импульсной струей еще более очевидно для аэродинамической эффективности, отношения подъемной силы к силе лобового сопротивления (рис. 3,a). В случае возбуждения модулированной импульсной струей при частоте модуляции $45 \mathrm{~Hz}$ увеличение подъемной силы достигает 13.1\% при угле атаки $0^{\circ}$, в то время как уменьшение силы лобового сопротивления достигает $20 \%$. В результате всего этого имеет место резкое увеличение отношения между подъемной силой и силой лобового сопротивления - на величину до $42 \%$ при нулевом угле атаки. При этом рост указанного отношения при возбуждении простой импульсной струей составляет около $36 \%$. Из рис. $3, b$ можно видеть, что в большинстве случаев возбуждения модулированной импульсной струей с частотой модуляции $45 \mathrm{~Hz}$ при одинаковых значениях подъемной силы величина силы лобового сопротивления оказывается ниже, чем в случае возбуждения простой импульсной струей.

В заключение отметим, что применение модуляции сигнала управления потоком имеет два достоинства. Во-первых, модуляция сигнала предоставляет возможность использовать одновременно две (высокую и низкую) частоты возбуждения для одной струи. Что касается области управления срывом потока, то значения этих двух различных частот возбуждения можно подобрать, основываясь на частотах двух доминирующих типов неустойчивых колебаний потока. Во-вторых, достоинство и примечательное качество метода модуляции сигнала состоит в том, что этим способом можно снизить расход воздуха по сравнению с расходом на простую импульсную струю за определенный период, в течение которого осуществляется управление потоком, при сохранении (и даже превышении) всех возможностей и достоинств, которые даются управлением.

\section{Конфликт интересов}

Авторы заявляют, что у них нет конфликта интересов. 


\section{Список литературы}

[1] A. Salmasi, A. Shadaram, A. Shams Taleghani, IEEE Trans. Plasma Sci., 41 (10), 3079 (2013). DOI: 10.1109/TPS.2013.2280612

[2] M. Mohammadi, A. Shams Taleghani, Arab. J. Sci. Eng., 39 (3), 2363 (2014). DOI: 10.1007/s13369-013-0772-1

[3] M. Sato, K. Okada, K. Asada, H. Aono, T. Nonomura, K. Fujii, Phys. Fluids, 32 (2), 025102 (2020). DOI: 10.1063/1.5136072

[4] А.В. Воеводин, А.А. Корняков, Д.А. Петров, А.С. Петров, Г.Г. Судаков, Письма в ЖТФ, 44 (15), 71 (2018). DOI: 10.21883/PJTF.2018.15.46443.17150 [A.V. Voevodin, A.A. Kornyakov, D.A. Petrov, A.S. Petrov, G.G. Sudakov, Tech. Phys. Lett., 44 (8), 687 (2018). DOI: $10.1134 / \mathrm{S} 106378501808014 \mathrm{X}]$.

[5] K.S. Aley, T.K. Guha, R. Kumar, AIAA J., 58 (5), 2053 (2020). DOI: 10.2514/1.J058939

[6] B. Steinfurth, F. Haucke, AIAA J., 56 (10), 3848 (2018). DOI: $10.2514 / 1 . J 057094$

[7] C. Cetin, A. Celik, M.M. Yavuz, AIAA J., 56 (1), 90 (2018). DOI: 10.2514/1.J056099

[8] C. Zhuang, G. Yang, Y. Zhu, D. Hu, Renew. Energy, 148, 964 (2020). DOI: 10.1016/j.renene.2019.10.082

[9] A. Saenz-Aguirre, U. Fernandez-Gamiz, E. Zulueta, A. Ulazia, J. Martinez-Rico, Sustainability, 11 (10), 2809 (2019). DOI: $10.3390 /$ su11102809

[10] H.Y. Xu, Q.L. Dong, C.L. Qiao, Z.Y. Ye, Energies, 11 (3), 619 (2018). DOI: 10.3390/en11030619

[11] M. Staats, W. Nitsche, S.J. Steinberg, R. King, CEAS Aeronaut. J., 8 (1), 197 (2017). DOI: $10.1007 / \mathrm{s} 13272-016-0232-1$

[12] R. Radespiel, M. Burnazzi, M. Casper, P. Scholz, Aeronaut. J., 120 (1223), 171 (2016). DOI: 10.1017/aer.2015.7

[13] D. Greenblatt, I.J. Wygnanski, Prog. Aerospace Sci., 36 (7), 487 (2000). DOI: 10.1016/S0376-0421(00)00008-7

[14] M.A. Feero, P. Lavoie, P.E. Sullivan, Exp. Fluids, 58 (8), 99 (2017). DOI: 10.1007/s00348-017-2387-x

[15] M. Amitay, A. Glezer, AIAA J., 40 (2), 209 (2002). DOI: $10.2514 / 2.1662$

[16] M. Amitay, D.R. Smith, V. Kibens, D.E. Parekh, A. Glezer, AIAA J., 39 (3), 361 (2001). DOI: 10.2514/2.1323

[17] A. Shams Taleghani, A. Shadaram, M. Mirzaei, S. Abdolahipour, J. Braz. Soc. Mech. Sci. Eng., 40 (4), 173 (2018). DOI: 10.1007/s40430-018-1120-x

[18] M. Mirzaei, A. Shams Taleghani, A. Shadaram, Appl. Mech. Mater., 186, 75 (2012).

DOI: 10.4028/www.scientific.net/AMM.186.75

[19] A. Shams Taleghani, A. Shadaram, M. Mirzaei, IEEE Trans. Plasma Sci., 40 (5), 1434 (2012). DOI: $10.1109 /$ TPS.2012.2187683

[20] A. Glezer, Phil. Trans.: Math. Phys. Eng. Sci., 369 (1940), 1476 (2011). http://www.jstor.org/stable/41061602 